Published in final edited form as:

Science. 2013 August 23; 341(6148): 849-850. doi:10.1126/science.1244156.

\title{
Mysterious Ribosomopathies
}

\author{
Kathleen L. McCann ${ }^{1}$ and Susan J. Baserga ${ }^{1,2,3}$ \\ Susan J. Baserga: susan.baserga@yale.edu \\ ${ }^{1}$ Department of Genetics, Yale University School of Medicine, New Haven, CT 06520, USA \\ 2Department of Molecular Biophysics and Biochemistry, Yale University School of Medicine, New \\ Haven, CT 06520, USA \\ ${ }^{3}$ Department of Therapeutic Radiology, Yale University School of Medicine, New Haven, CT \\ 06520, USA
}

\section{Abstract}

Ribosomes are essential in all cell types, yet mutations to ribosomal proteins or assembly factors cause tissue-specific disease.

\begin{abstract}
Ribosomes are absolutely essential for life, generating all cellular proteins required for growth. The prevailing thought for many years was that mutations in ribosomal proteins or ribosome assembly factors would be lethal to developing embryos. Complete loss of any single ribosomal protein often leads to embryonic lethality in mice (1). Yet, mutations in ribosomal proteins or ribosome assembly factors result in a puzzling phenomenon-a specific mutation can affect a specific cell type and cause a tissue-specific human disease. What accounts for this tissue proclivity has been a mystery. Why do defects in a macromolecule as ubiquitous and essential as the ribosome cause diseasesribosomopathies-only in select tissues?
\end{abstract}

Eukaryotic ribosomes are large, intricate cellular machines that translate messenger RNA (mRNA) into protein. They comprise four different ribosomal RNAs (rRNAs; $18 S$ in the small subunit and $28 S, 5.8 S$, and $5 S$ in the large subunit), as well as $\sim 80$ ribosomal proteins (see the figure). More than 200 assembly factors and small RNAs are needed to synthesize ribosomes in the nucleolus. Some of the structural components also control the processing of precursor ribosomal RNA (pre-rRNA), assembly of the ribosomal subunits, and the translation process itself. Over the past 15 years, mutations in ribosomal proteins or ribosome biogenesis factors have been found in patients with varying diseases. At least $50 \%$ of patients with Diamond-Blackfan anemia carry mutations in ribosomal proteins (2). Other ribosomopathies identified include Treacher-Collins syndrome, North American Indian childhood cirrhosis, chromosome 5q-syndrome $(3,4)$, and isolated congenital asplenia (5).

With the exception of 5q-syndrome, ribosomopathies are congenital, indicating that they impair development, but this impairment is not universal among all tissues or organs. For example, in isolated congenital asplenia, the most recently discovered ribosomopathy, haploinsufficiency (having only one functional copy of a gene, with the other copy inactivated by mutation) of the ribosomal protein RPSA prevents splenic development. These patients are prone to severe bacterial infections because they lack a spleen, but they are otherwise healthy and have no other observable and have no other observable developmental anomalies (5). This is surprising because RPSA is a component of the small subunit of the ribosome and is thus ubiquitously expressed. The mutation in RPSA is present in all tissues, not just the spleen, so one might expect haploinsufficiency of RPSA to affect development of all tissues, not just the spleen. 
In the case of Diamond-Blackfan anemia, mutations in any of 11 different ribosomal proteins lead to bone marrow failure. The haploinsufficiencies can affect pre-rRNA processing and ribosome assembly, but not the same steps. In North American Indian childhood cirrhosis, a mutation in the ribosome biogenesis factor hUTP4/Cirhin causes biliary cirrhosis, for which the only treatment is liver transplantation required by early adolescence. Little is known about the molecular mechanisms that lead to this disease. Shwachman-Bodian-Diamond syndrome arises from mutations in the SBDS protein, which is involved in large ribosomal subunit maturation. Patients with this disorder suffer not only from dysfunction of the pancreas, but also from bone marrow failure, skeletal abnormalities, and an enlarged liver. Although there are some shared defects among many of the different ribosomopathies (e.g., bone marrow failure, skeletal abnormalities, growth retardation, and increased risk of cancer), these diseases are clinically distinct and require different therapeutic interventions.

One possible explanation for the conundrum of ribosomopathies is that the affected tissues are rapidly dividing and therefore are highly sensitive to mutations that might reduce the number of ribosomes. It is true that bone marrow cells need large numbers of ribosomes to make proteins, grow in size, and divide. However, this cannot be the full explanation, as all organ systems in a developing embryo are rapidly dividing and therefore require a large number of ribosomes.

An alternative explanation is that the composition of ribosomes may be different in different cell types, which could account for the various effects of ribosomal protein haploinsufficiency. This idea may contradict the notion that ribosomes are monolithic machines, but it is not completely unfounded. Surprisingly, the reduction of a specific ribosomal protein can lead to changes in the spectrum of translated mRNAs without affecting overall protein synthesis. For example, when the ribosomal protein L38 (Rpl38) is depleted, a subset of mRNAs that encode homeobox genes are not translated during mouse embryogenesis (6). Likewise, reduction of Rpl40 impairs translation of vesicular stomatitis virus mRNAs in cultured human cells (7). Indeed, the amounts of mRNA that encode ribosomal proteins also vary among tissues in the developing mouse embryo (6). It is not clear whether these differences are reflected in the constituents of the functioning ribosome. If so, this raises the question of "specialized" ribosomes, and one must look closely for whether ribosome composition influences the pathogenesis of ribosomopathies.

Another possible explanation for tissue proclivity is that mutations in ribosomal proteins or assembly factors result in reduced numbers of fully functional cytoplasmic ribosomes. Although ribosome amounts would be adequate for survival, a smaller number of ribosomes could alter the mRNAs that are translated. This may, in turn, inhibit normal cellular growth and differentiation in specific cell types. In Diamond-Blackfan anemia, a mutation in Rps19 results in defects in the processing of the pre- $18 S$ rRNA, thereby reducing the amount of the small subunit that is assembled $(1,2)$. Indeed, in mouse erythroblasts with reduced amounts of Rps19, translation of a subset of mRNAs is decreased (8). Other ribosomopathies with proposed nucleolar dysfunction, such as North American Indian childhood cirrhosis, may similarly result in reduced amounts of ribosomal subunits (9).

Additionally, in some cases, mutations that disrupt ribosome assembly can cause the nucleolar stress response in some cell types. This response involves increased synthesis of the protein p53. This tumor suppressor protein arrests cell division in response to such stress, which leads to programmed cell death (apoptosis) (10). In a mouse model for Treacher-Collins syndrome, craniofacial dysmorphology was rescued when p53 expression was reduced (11). Treacher-Collins syndrome often results from haploinsufficiency in TCOF 1, the gene that encodes Treacle, a nucleolar protein involved in pre-rRNA synthesis. 
Similarly, in mouse (haploinsufficiency in Rps19) and zebrafish (haploinsufficiency in Rps29) models for Diamond-Blackfan anemia, p53 inactivation rescued some of the red cell lineage abnormalities (12). However, in a zebrafish model for Shwachman-Bodian-Diamond syndrome, loss of p53 did not rescue the associated exocrine pancreas developmental defects (13). Do some cell types more readily succumb to nucleolar stress? Activation of p53 may contribute to the tissue proclivity of some ribosomopathies, but perhaps not all.

Mutations in ribosomal proteins and assembly factors confer a broad clinical spectrum. Although the mechanisms underlying the tissue proclivity in ribosomopathies are yet to be defined, these disorders do make it increasingly clear that the days of thinking of ribosomes as unchanging monoliths are coming to an end.

\section{Acknowledgments}

Supported by NIH GM 52581 .

\section{References and Notes}

1. McGowan KA, et al. Nat Genet. 2008; 40:963. [PubMed: 18641651]

2. Raiser DM, Narla A, Ebert BL. Leuk Lymphoma. 2013; 1

3. Freed EF, Bleichert F, Dutca LM, Baserga SJ. Mol Biosyst. 2010; 6:481. [PubMed: 20174677]

4. Burwick N, Shimamura A, Liu JM. Semin Hematol. 2011; 48:136. [PubMed: 21435510]

5. Bolze A, et al. Science. 2013; 340:976. [PubMed: 23579497]

6. Kondrashov N, et al. Cell. 2011; 145:383. [PubMed: 21529712]

7. Lee AS, Burdeinick-Kerr R, Whelan SPJ. Proc Natl Acad Sci USA. 2013; 110:324. [PubMed: 23169626]

8. Horos R, et al. Blood. 2012; 119:262. [PubMed: 22058113]

9. Freed EF, Prieto JL, McCann KL, McStay B, Baserga SJ. PLoS Genet. 2012; 8:e1002892. [PubMed: 22916032]

10. Rubbi CP, Milner J. EMBO J. 2003; 22:6068. [PubMed: 14609953]

11. Jones NC, et al. Nat Med. 2008; 14:125. [PubMed: 18246078]

12. Boultwood J, et al. Adv Enzyme Regul. 2012; 52:196.

13. Provost E, et al. Development. 2012; 139:3232. [PubMed: 22872088] 


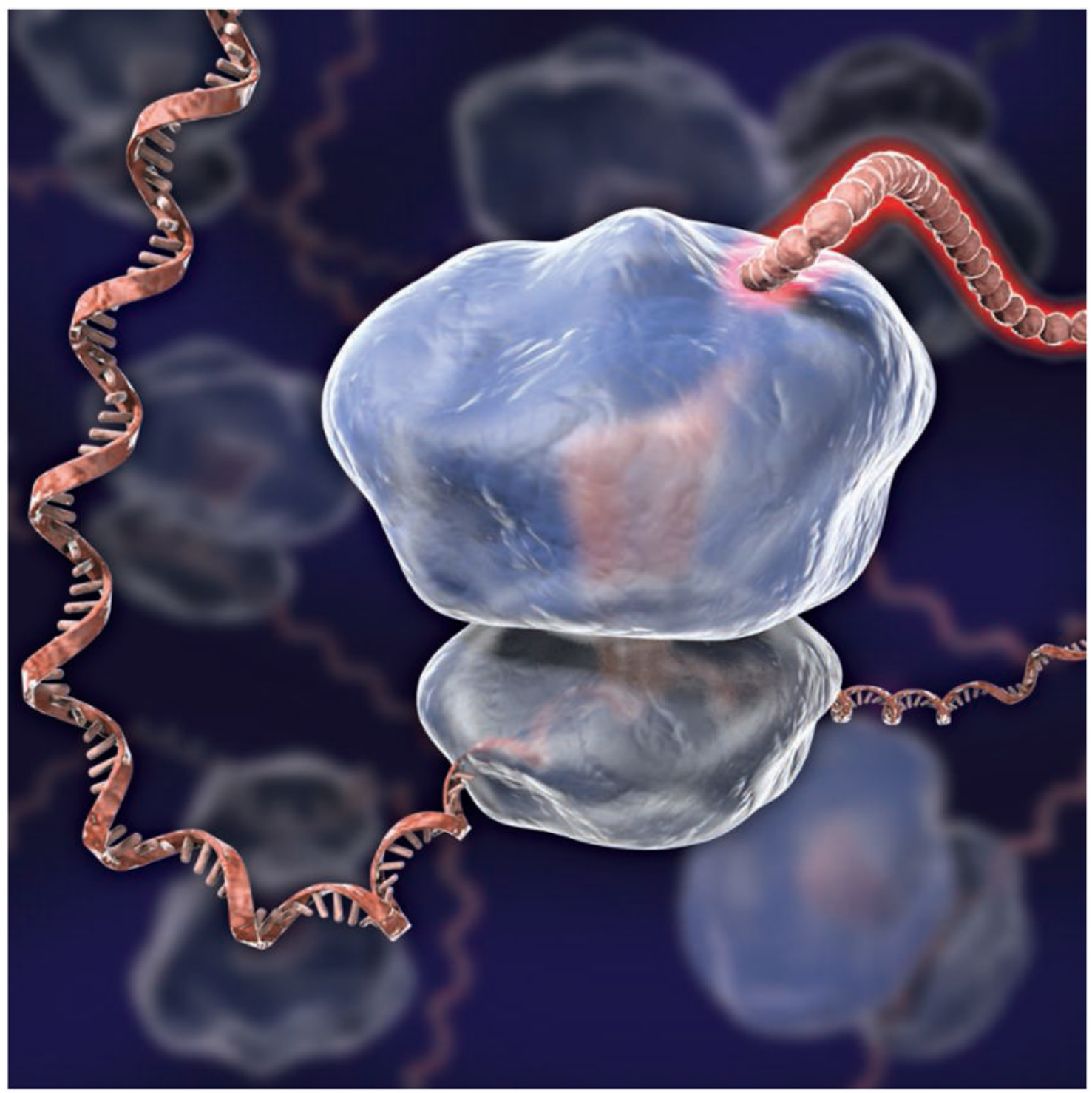

Figure. Monolith no longer

Mutations in ribosomal proteins or ribosome biogenesis factors have been found in patients with varying tissue-specific diseases. 